\title{
Kosteneffiziente Reinigung von Abluft
}

\author{
Mit weiterentwickelten Abluftreinigungsanlagen lassen sich die Energie- und Wartungskosten \\ sowie der Wartungsaufwand deutlich reduzieren. Die Standzeiten verlängern sich deutlich bei \\ gleichzeitig hoher Energieeffizienz.
}

$D^{\text {it }}$ e Reinigung von Abluft steht im Spannungsfeld von komplexen Schadstoffgemischen (Bild 1) und steigenden Anforderungen an Energieeffizienz, Verfügbarkeit und Flexibilität. Insbesondere die Möglichkeit, Standzeiten zu erhöhen sowie die Wartungskosten und die Energiekosten zu senken, stellt einen klaren Wettbewerbsvorteil für Betreiber von Abluftreinigungsanlagen dar. So ist es das Ziel vieler Unternehmen, robuste, unkomplizierte, energieeffiziente Anlagen einzusetzen, um Aufwand und Kosten nachhaltig zu reduzieren.

Siliziumorganische Verbindungen in Abluftströmen verursachen in thermischen Anlagen zur Abluftreinigung Schwierigkeiten, da aus den gasförmigen Substanzen im Brennraum zwangsläufig der Feststoff $\mathrm{SiO}_{2}$ entsteht. Dieser Zusammenhang besteht bei der thermisch-regenerativen Nachverbrennung (RNV), der thermisch-rekuperativen Nachverbrennung (TNV) und auch der katalytischen Nachverbrennung (KNV).

Anlagenstillstand durch $\mathrm{SiO}_{2}$ vermeiden

Der Oberbegriff „Siliziumorganische Verbindungen" fasst folgende Stoffgruppen zusammen: Silane, Siloxane und Silikone. Diese treten beispielsweise bei Abluftströmen aus Reifenherstellungsprozessen oder bei der Verwendung von Silikonlacken auf. Insbesondere Silane, die häufig als Haftvermittler eingesetzt werden, finden immer breitere Verwendung in Lacksystemen.

Bereits bei sehr geringen Konzentrationen kann das entstehende $\mathrm{SiO}_{2}$ lokal akkumulieren und so sukzessive Leitungen, Wärmetauscherrohre und Wärmespeichersteine verschließen sowie Katalysatoren deaktivieren. Dadurch verschlechtern sich die Reingaswerte und die Druckverluste erhöhen sich. Das führt zu Anlagenstillständen und zieht neben dem Aufwand für außerplanmäßigen Betriebsstillstand auch hohe Wartungskosten nach sich.

Die Technologie zur thermisch regenerativen Nachverbrennung (RNV) ist bereits für ein sehr breites Spektrum an Schadstoffen konzipiert. Die Anlagen arbeiten durch die integrierte Wärmerückgewinnung mit geringem oder ohne zusätzlichen Brennstoff (Bild 2).

Der Anlagenhersteller Rotamill hat das Portfolio hierzu weiterentwickelt. Die umfangreichen Erfahrungen mit zahlreichen RNV-Anlagen, die Abluft mit siliziumorganischen Verbindungen reinigen, sind in ein neues RNV-internes $\mathrm{SiO}_{2}$-Management geflossen. Das erlaubt nun deutlich längere Standzeiten mit siliziumorganischen Verbindungen in der Abluft bei gleichzeitig gewohnt hoher Energieeffizienz der RNV-Technologie. Zudem werden die Wartungskosten im Vergleich zu Anlagen ohne $\mathrm{SiO}_{2}$-Management deutlich reduziert. I

\section{Hannover Messe, Halle 3, Stand B08}

\section{Kontakt:}

Daniel Hein, Eberhard Krumm, Verfahrenstechnischer Anlagenbau Rotamill Anlagen-, Apparate- und Ventilatorenbau, Siegen, Tel.0271 66123-325, d.hein@rotamill.de, www.rotamill.de
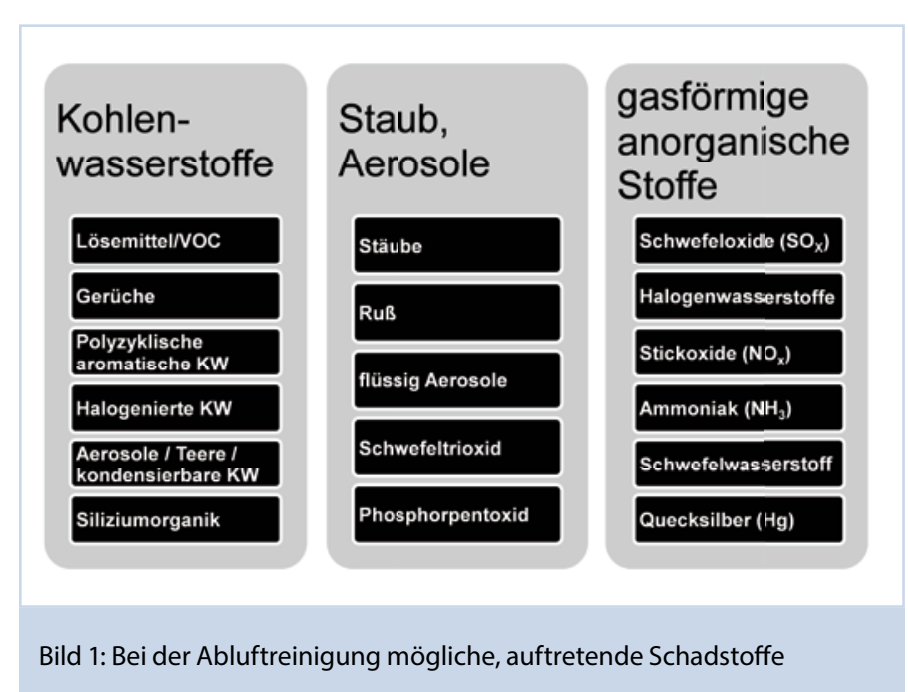

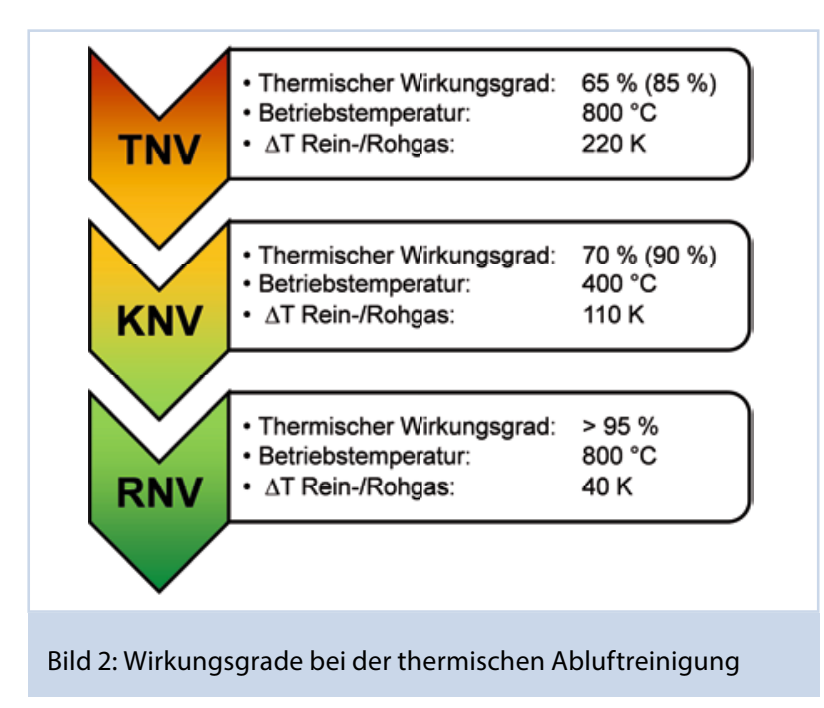

